\title{
The Effectiveness of Emotional Working Memory Training on Beta Asymmetry in Frontal Regions of Two Hemispheres in People with Borderline Personality Disorder
}

\author{
Sajjad Basharpoor $^{1 *}$, Nasim Zakibakhsh Mohammadi ${ }^{1}$, Mohammad Narimani ${ }^{1}$, Moslem Kord ${ }^{2}$ \\ ${ }^{1}$ Department of Psychology, University of Mohaghegh Ardabili, Ardabil, Iran \\ ${ }^{2}$ Research Center of Cognitive Sciences, Department of Cognitive Sciences, Shahid Beheshti University, Tehran, Iran
}

Article Info:

\section{A BSTRACT}

Introduction: The prevalence of borderline personality disorder is more than other type of personality disorders. It has been shown that there are some neurological deficits in patients with borderline personality disorder such as cognitive inhibition and self-injury, but the few studies aimed to decrease this deficit have been conducted. The purpose of this study was to investigate the effectiveness of emotional working memory training on the EEG asymmetry index in the beta band between two hemispheres in people with borderline personality disorder. Materials and Methods: The method of the current study was experimental and its design was pretest-posttest with the control group. All students of the University of Mohaghegh Ardebili with borderline personality disorder in 96-97 academic years comprised the statistical population of this study. Forty people selected by screening method via scale of borderline personality disorder trait and the structural clinical interview for mental disorders (SCID-II) and assigned to two experimental and control groups. The experimental group received 10 sessions of emotional working memory training, but no intervention was provided for the control group. The EEG device was used to record the electrical activity of the brain from the frontal lobe (Fp1, Fp2, F3, F4, F7, F8). Results: The results showed that the mean scores of the interhemispheric beta asymmetry have increased in the experimental group compared to the control group at the post-test stage. The emotional working memory training can lead to increased interhemispheric beta asymmetry. This intervention can increase the beta asymmetry by enhancing the beta wave in left hemisphere rather than right. Conclusion: the emotional working memory training can be the main axis to improve interhemispheric beta asymmetry in people with borderline personality disorder.

\section{Key words:}

1. Borderline Personality Disorder

2. Patients

3. Electroencephalography

*Corresponding Author: Sajjad Basharpoor

E-mail: basharpoor_sajjad@uma.ac.ir 


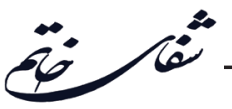

اثربخشى آموزش حافظةٌ كارى هيجانى بر عدم تقارن بتا در بين مناطق ييشانى دو نيمكرهُ مغزى افراد

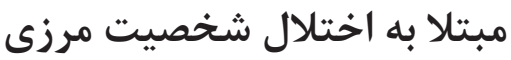

\author{
سجاد بشر يور '."، نسيم ذكىبخش محمدى'، محمد نريمانى'، مسلم كرد׳ \\ 'اكروه روانشناسى، دانشخاه محقق اردبيلى، اردبيل، ايران

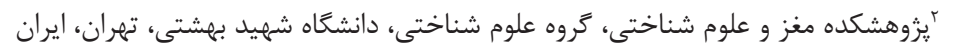

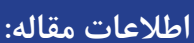

بذيرش: ب آذر גهץ|

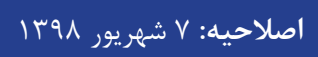

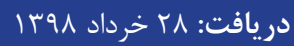

كليد وازهها:

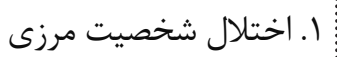

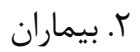

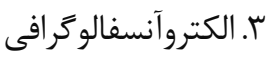

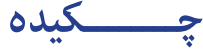

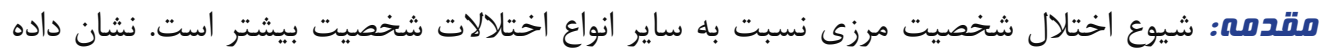

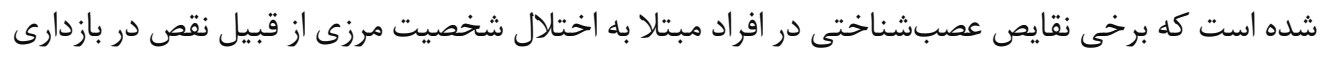

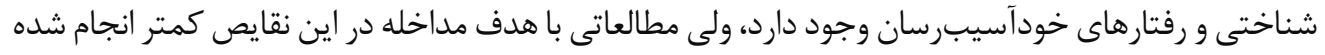

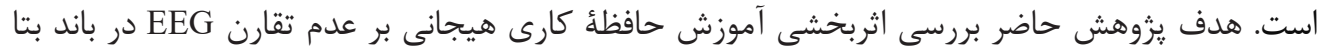

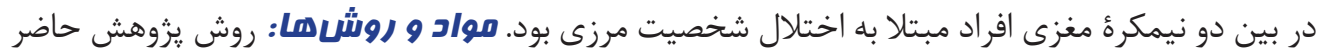

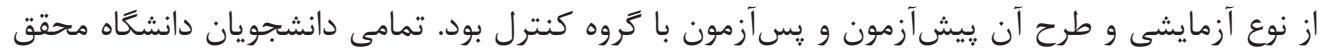

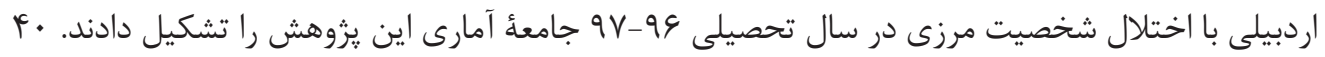

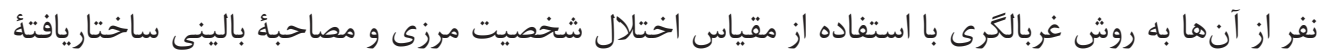

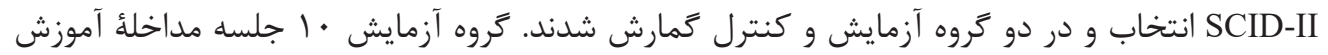

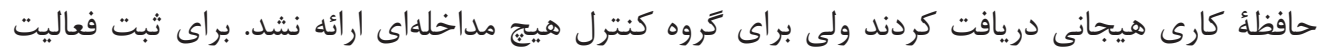

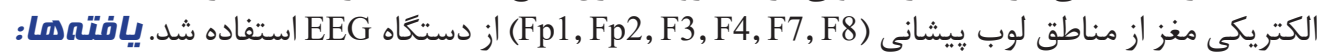

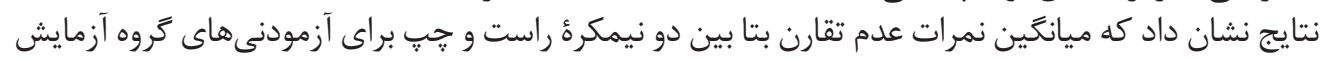

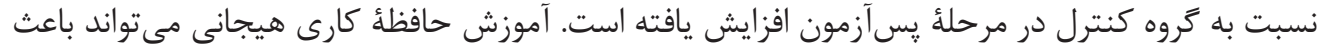

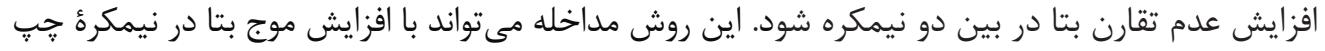

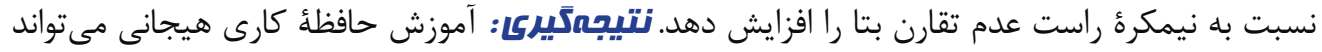

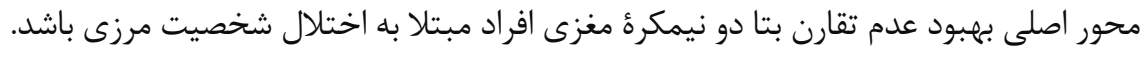

" آدويسنده مسئول: سجاد بشريور

آدرس الكترونيكى: basharpoor_sajjad@uma.ac.ir 


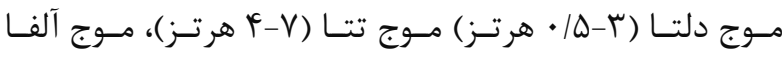

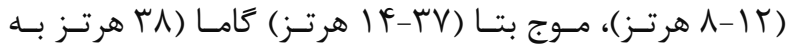

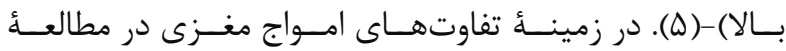

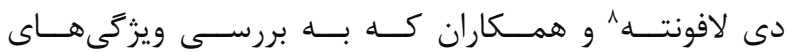

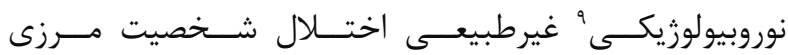

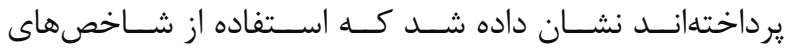
EEG

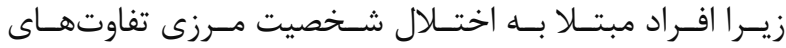

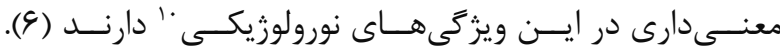

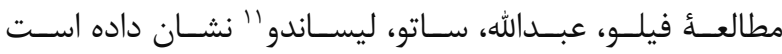

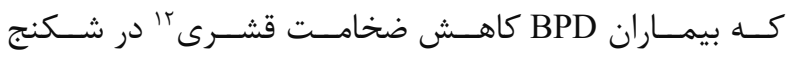

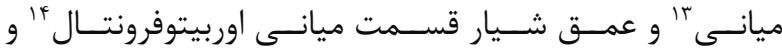

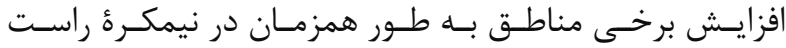

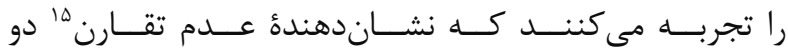

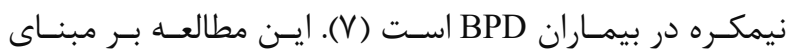

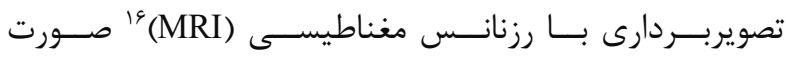

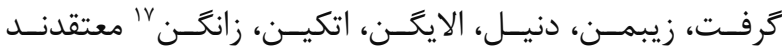

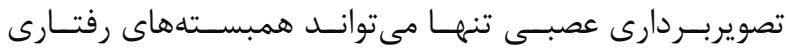

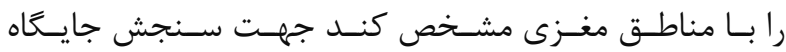

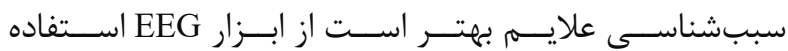

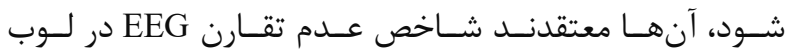

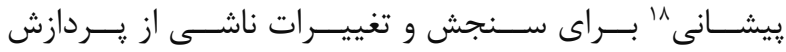

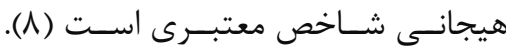

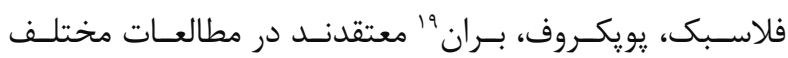

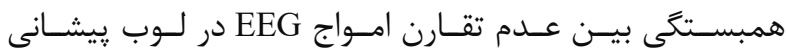

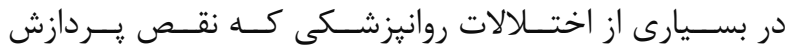

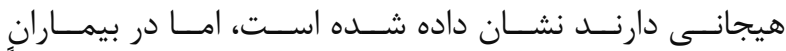

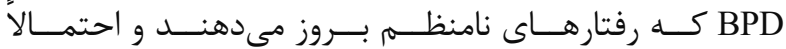

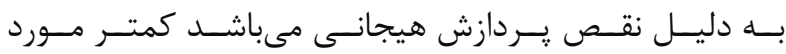

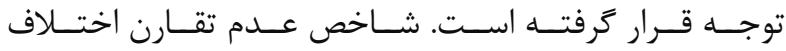

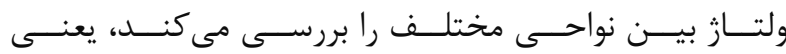

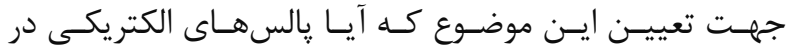

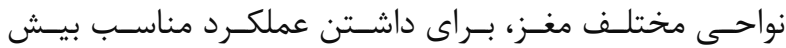

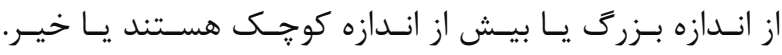

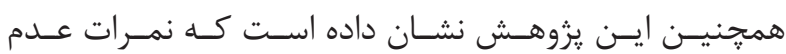

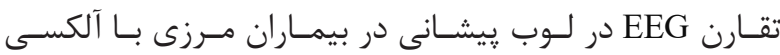

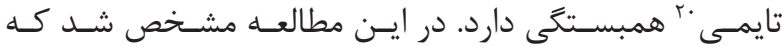

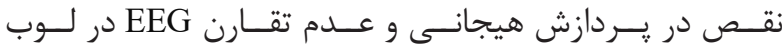

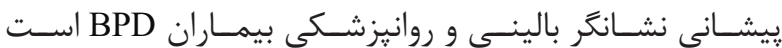

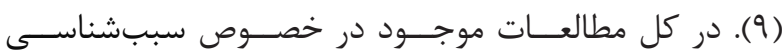

${ }^{1}$ Borderline personality disorder

${ }^{2}$ Diagnostic and statiscal manual of mental disorder (Fifth Edition)

${ }^{3}$ Interpersonal relationships

${ }^{4}$ Self-concept

${ }^{5}$ Impulsivity

${ }^{6}$ Andrulonis

${ }^{7}$ Borderline personality disorder

${ }^{8}$ De la Fuente

${ }^{9}$ Neurobiological

${ }^{10}$ Neurological

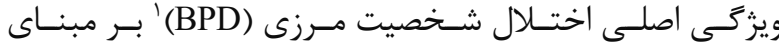

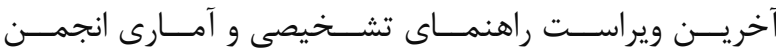

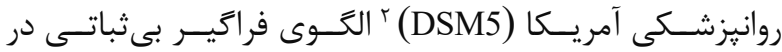

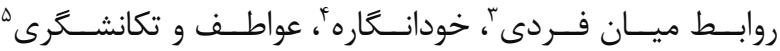

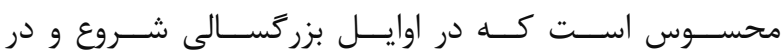

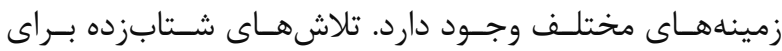

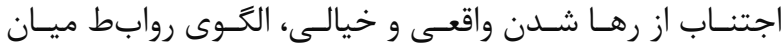

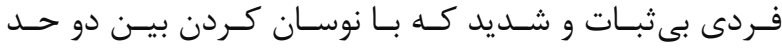

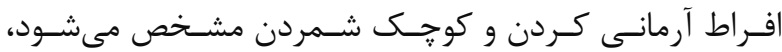

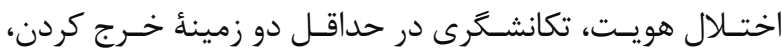

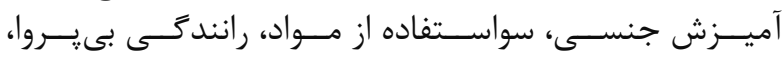

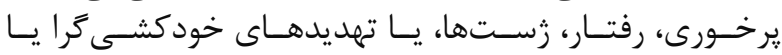

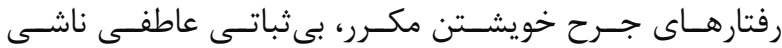

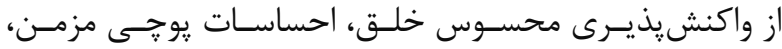

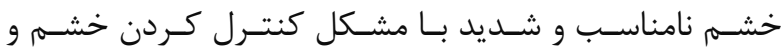

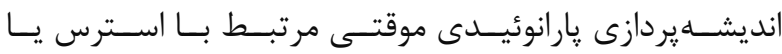

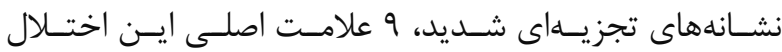

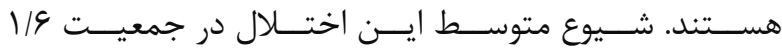

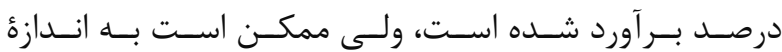

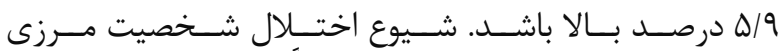

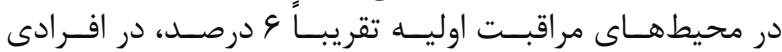

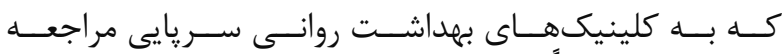

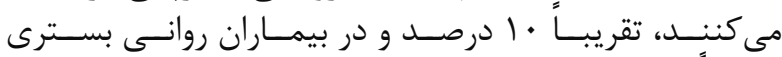

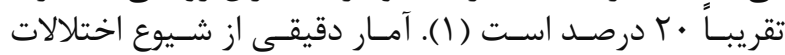

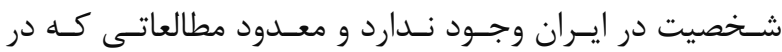

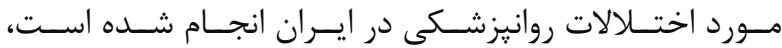

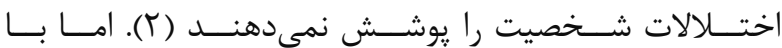

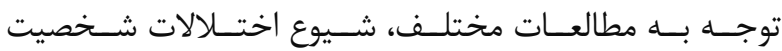

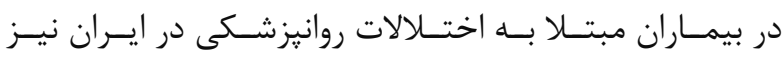

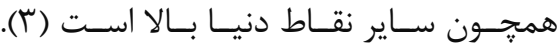

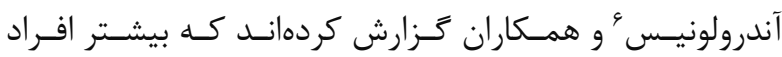

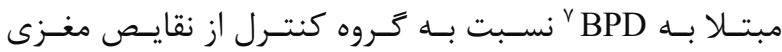

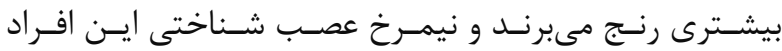

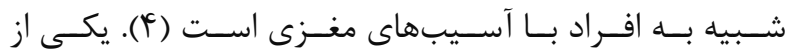

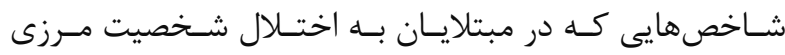

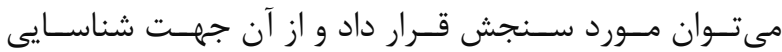

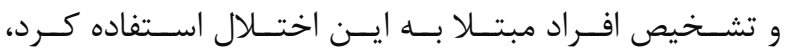

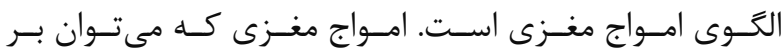

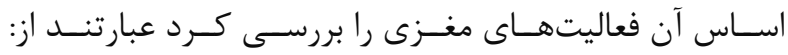

${ }^{11}$ Filho, Abdallah, Sato, and Lisondo

${ }^{12}$ Cortical thickness

${ }^{13}$ Mean curvature

${ }^{14}$ Orbitofrontal

${ }^{15}$ Asymmetry

${ }^{16}$ Magnetic resonance imaging

${ }^{17}$ Zibman, Daniel, Alyagon, Etkin, and Zangen

${ }^{18}$ Frontal

${ }^{19}$ Flasbeck, Popkirov, and Brüne

${ }^{20}$ Alexithymia 


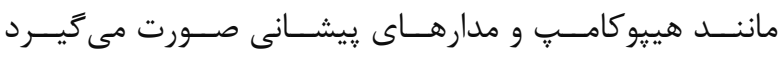

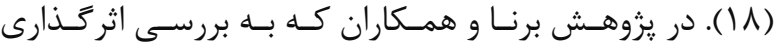

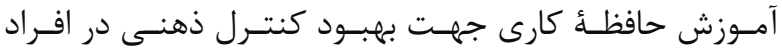

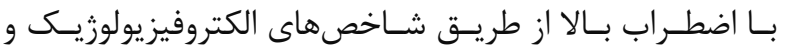

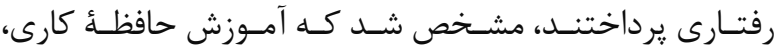

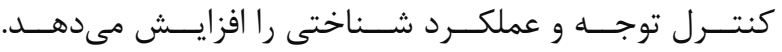

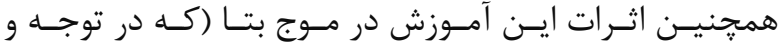

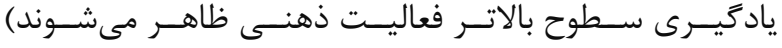

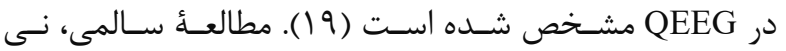

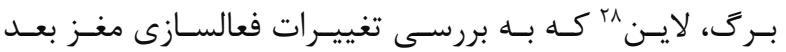

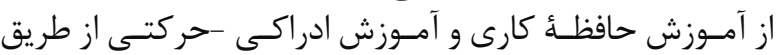

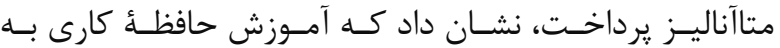

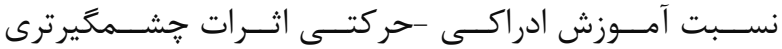

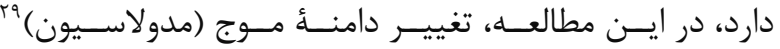

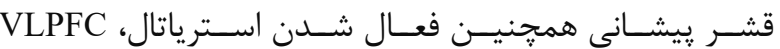

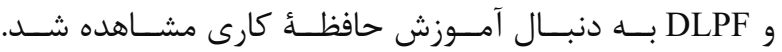

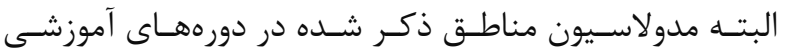

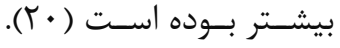

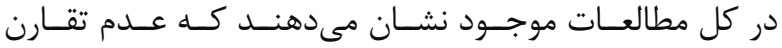

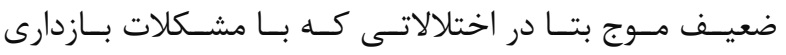

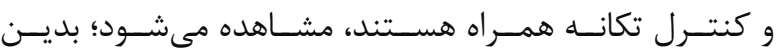

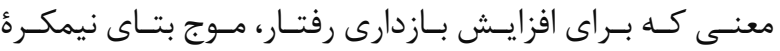

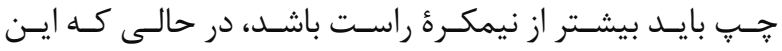

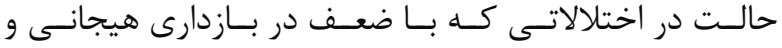

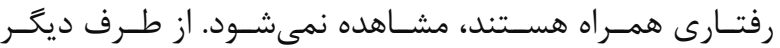

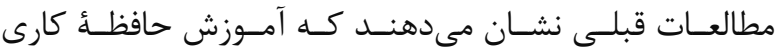

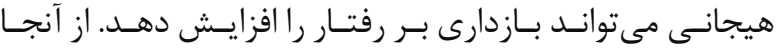

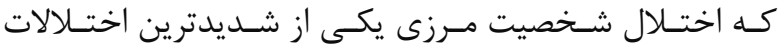

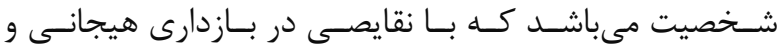

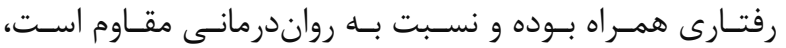

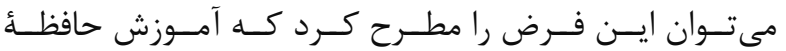

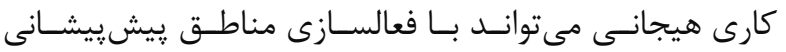

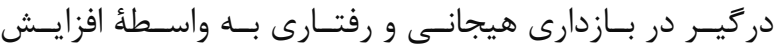

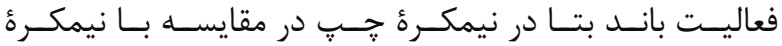

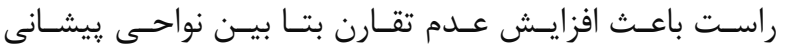

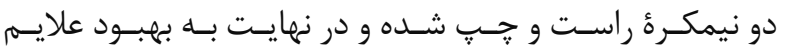

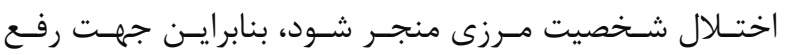

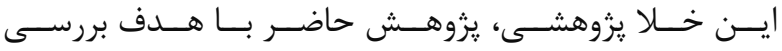

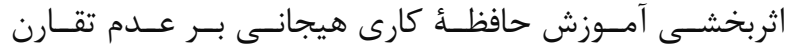

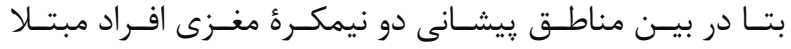

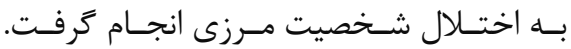

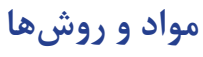
جامعةُ آمارى و نمونه

\footnotetext{
${ }^{21}$ Frontolimbic

${ }^{22}$ Emotional working memory training

${ }^{23}$ Frontoparietal

${ }^{24}$ Dorsolateral prefrontal

${ }^{25}$ Inferior parietal
}

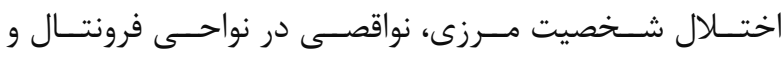

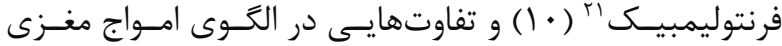

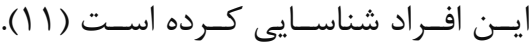

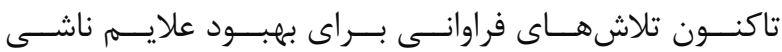

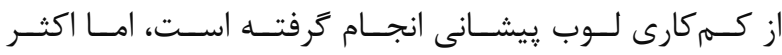

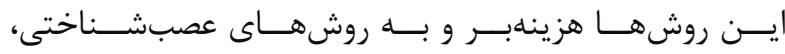

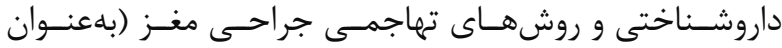

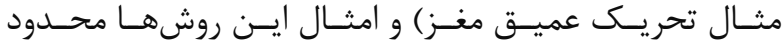

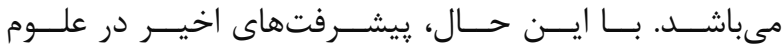

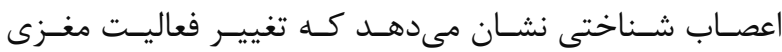

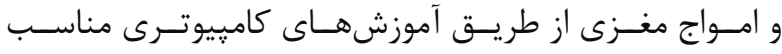

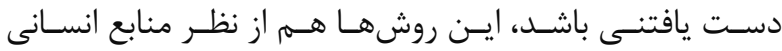

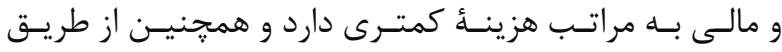

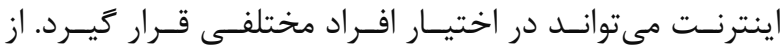

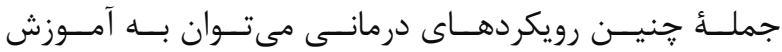

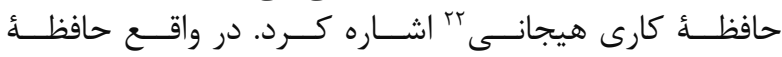

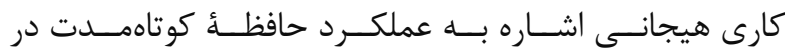

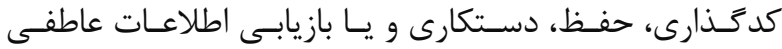

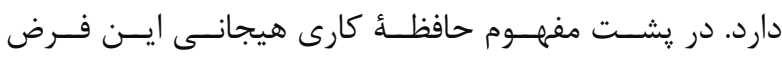

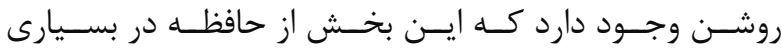

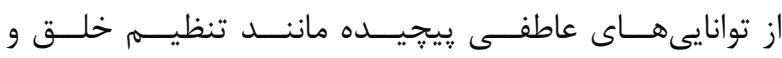

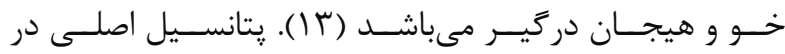

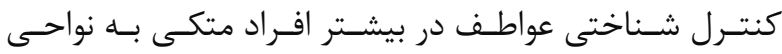

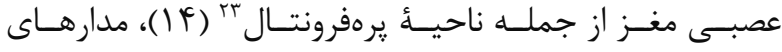

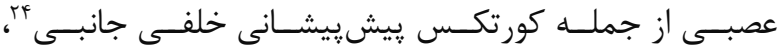

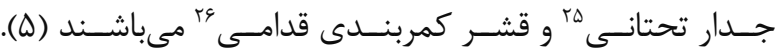

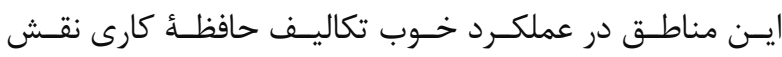

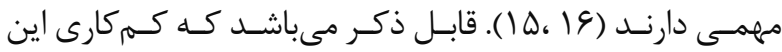

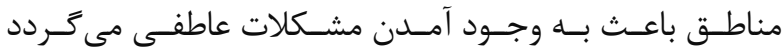

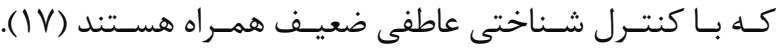

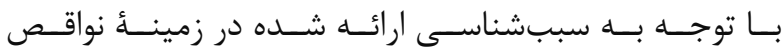

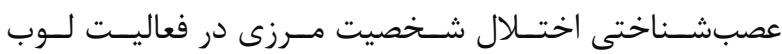

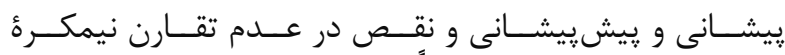

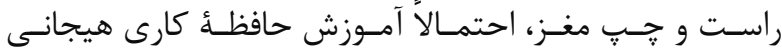

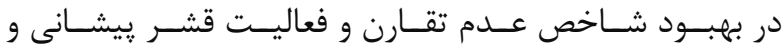

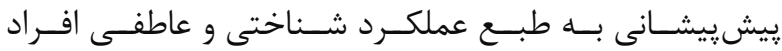

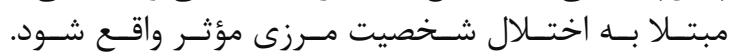

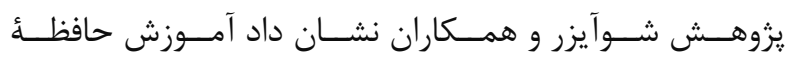

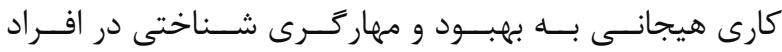

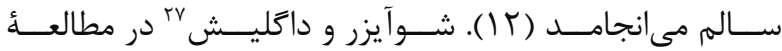

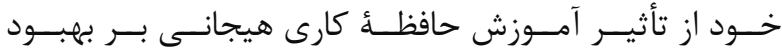

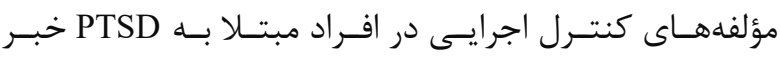

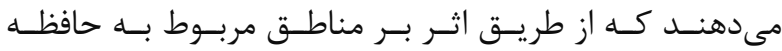

${ }^{26}$ Anterior cingulate

${ }^{27}$ Dalgleish and SchweIzers

${ }^{28}$ Salmi, Nyberg, and Laine

${ }^{29}$ Modulation 


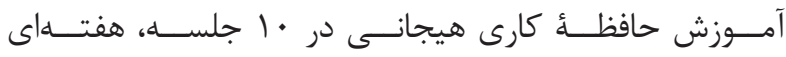

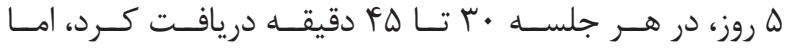

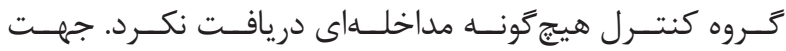

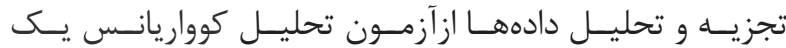

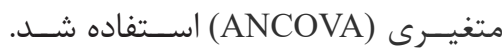

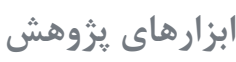

\section{الف - مقياس شخصيت مرزى}

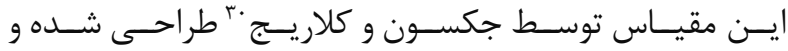

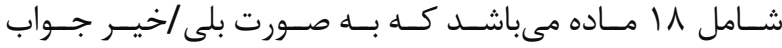

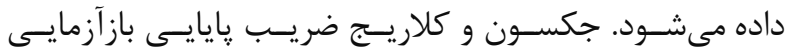

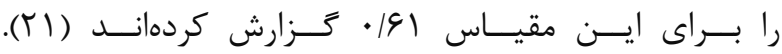

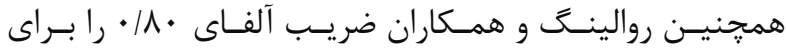

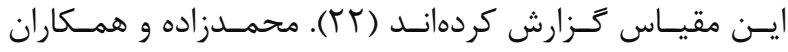

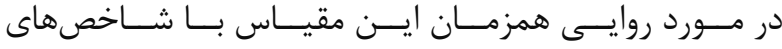

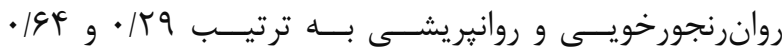

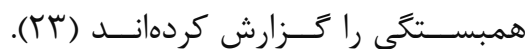

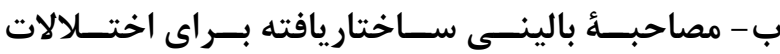

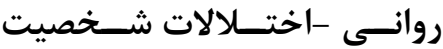

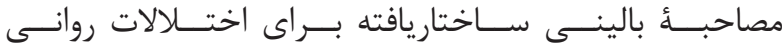

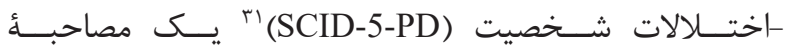

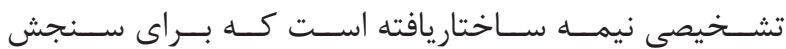

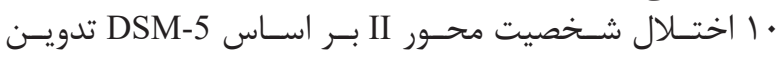

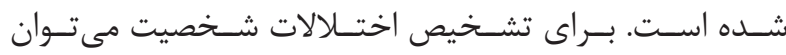

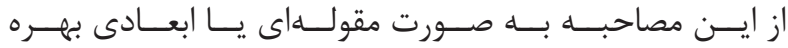

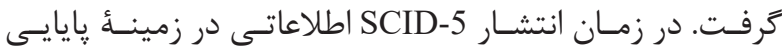

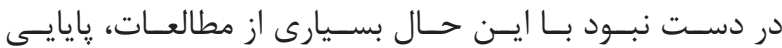

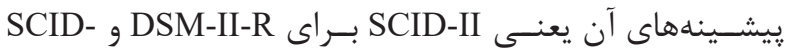

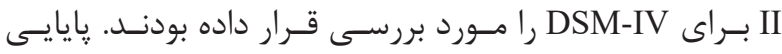

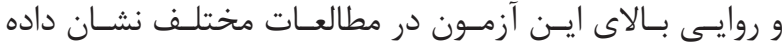

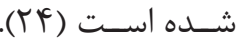

ج - دســتَاه ثبــت امــواج مغـزى (الكتروانســفالوكر افىى

(EEG

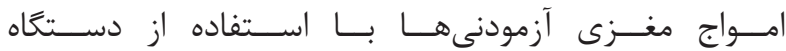

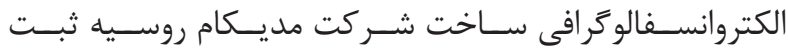

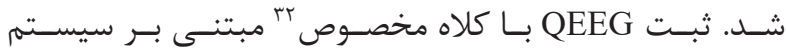

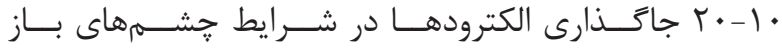

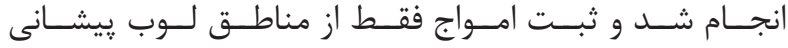

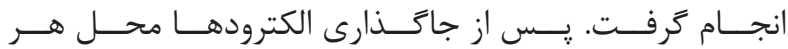

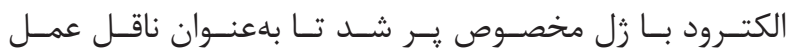

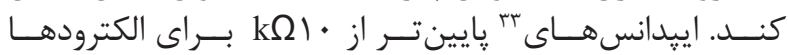

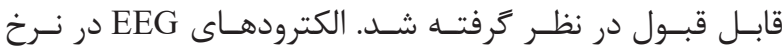

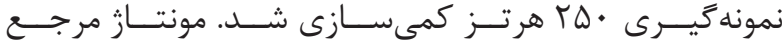
linked ears

${ }^{30}$ Jackson and Claridge

${ }^{31}$ Structured clinical interview for DSM-5 personality

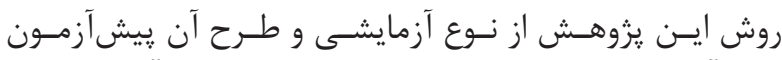

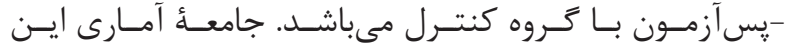

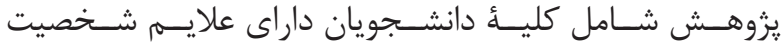

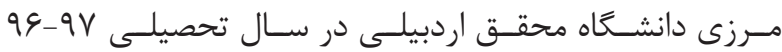

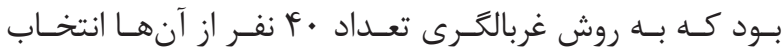

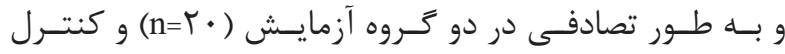

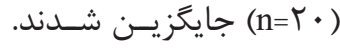

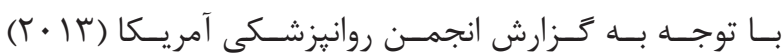

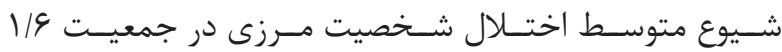

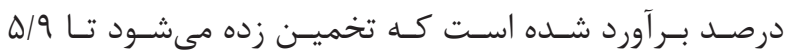

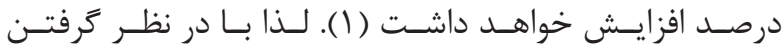

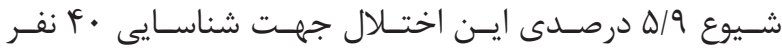

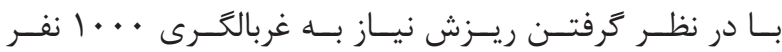

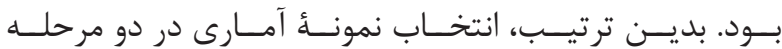

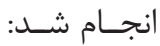

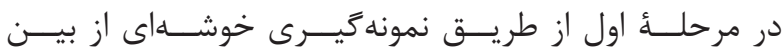

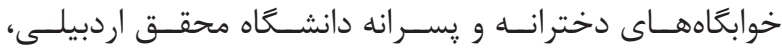

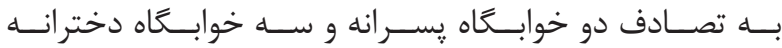

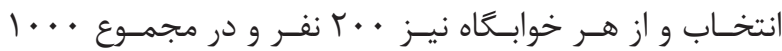

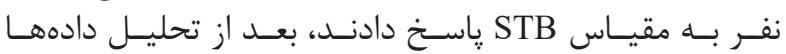

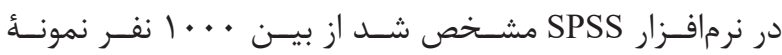

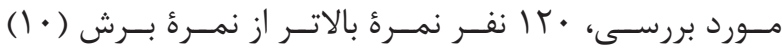

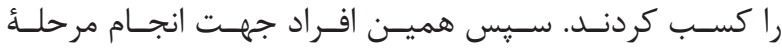

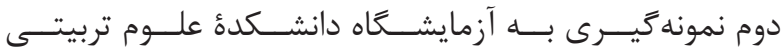

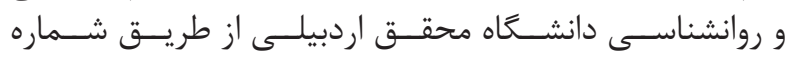

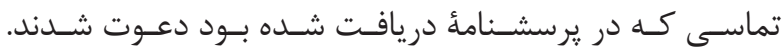

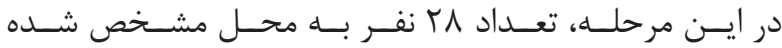

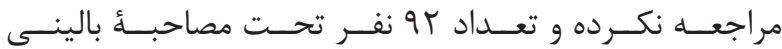

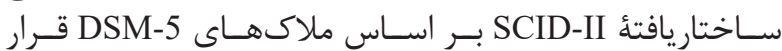

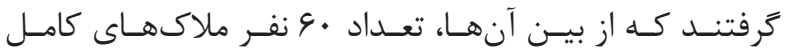

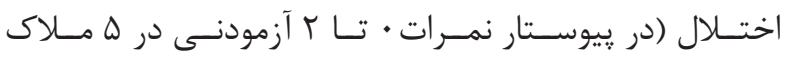

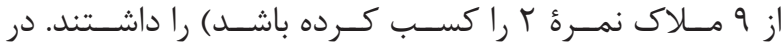

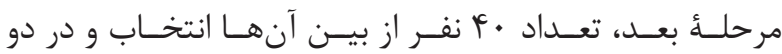

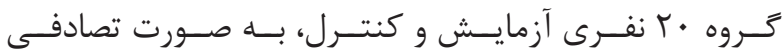

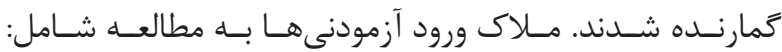

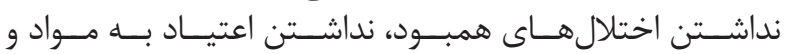

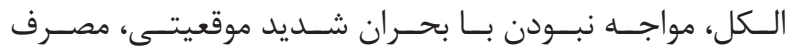

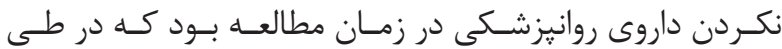

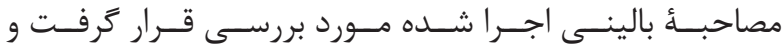

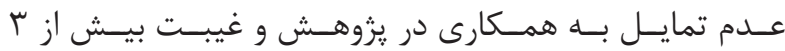

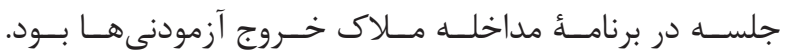

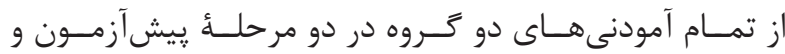

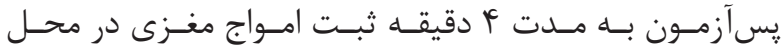

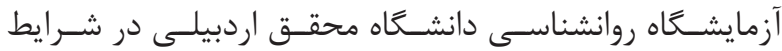

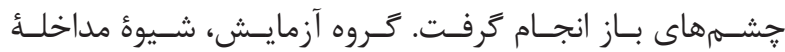

${ }^{32}$ Electro cap

${ }^{33}$ Electrode impedances 


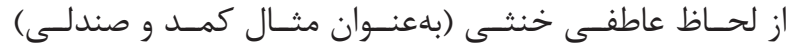

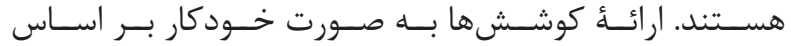

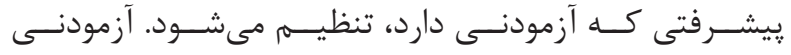

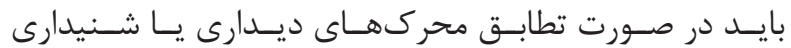

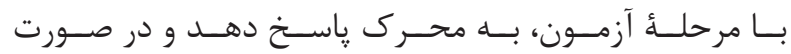

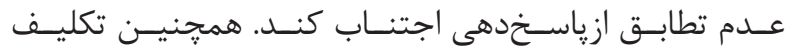

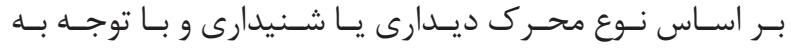

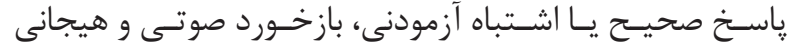

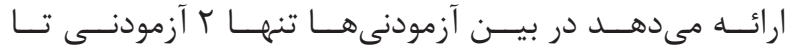

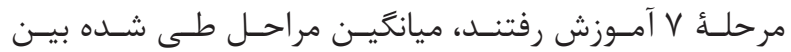

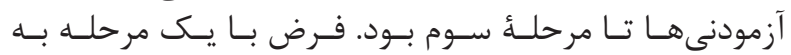

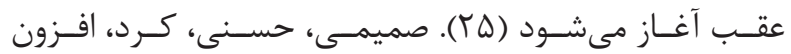

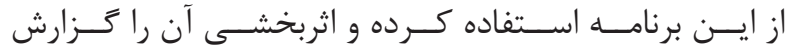

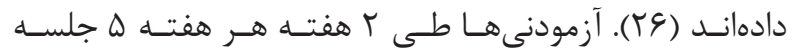

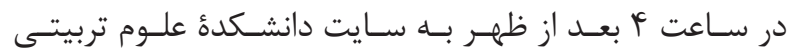

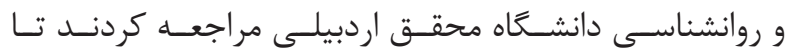

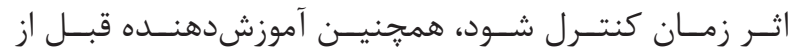

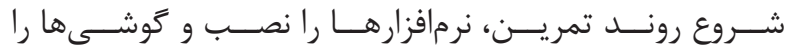

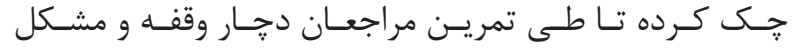

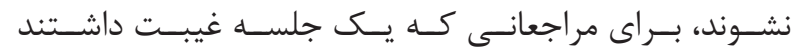

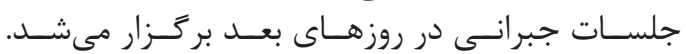

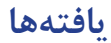

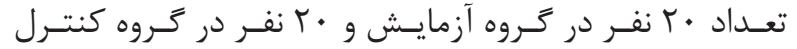

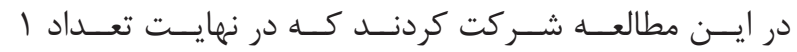

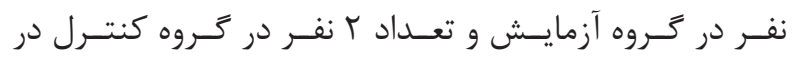

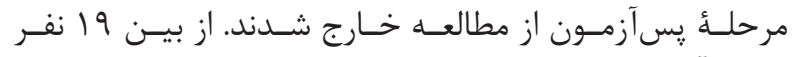

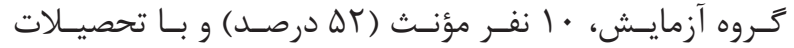

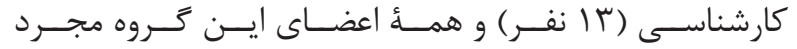

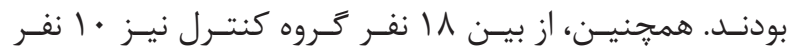

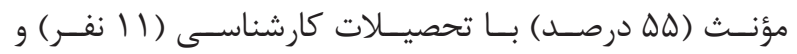

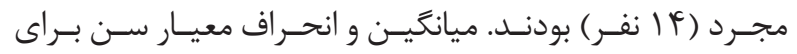

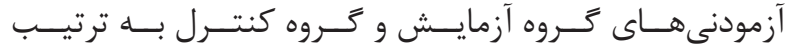
. بـود

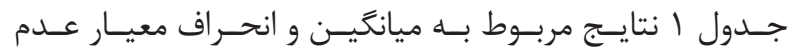

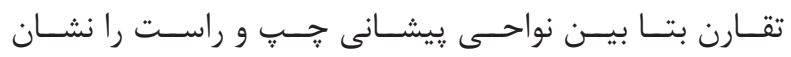
مى دهــد

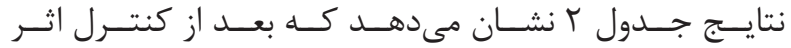

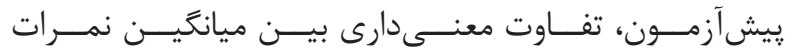

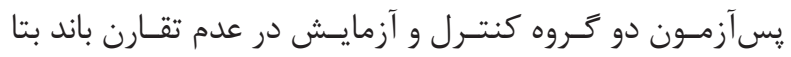

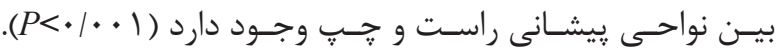

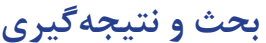

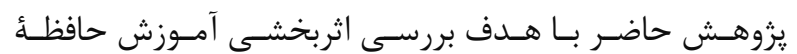

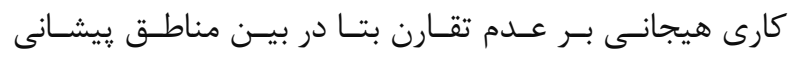

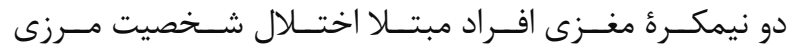

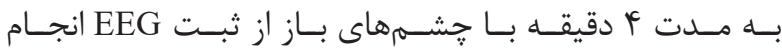

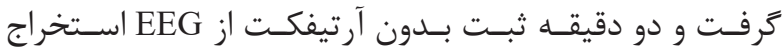

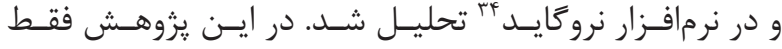

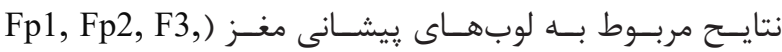
(F4, F7, F8

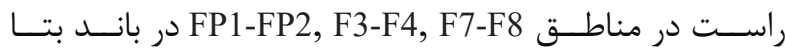

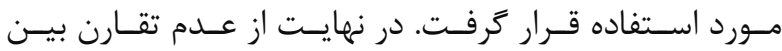

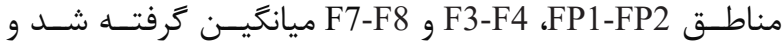

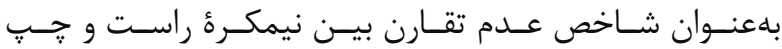

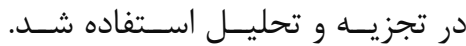

\section{د- نرمافزار آموزش حافظهُ كارى هيجانى}

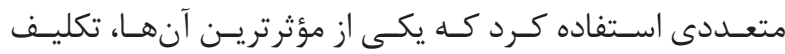

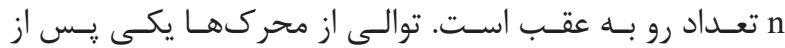

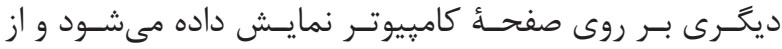

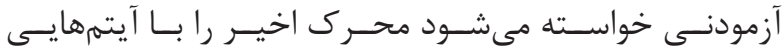

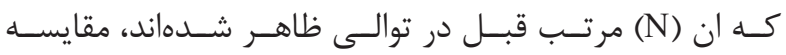

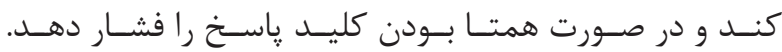

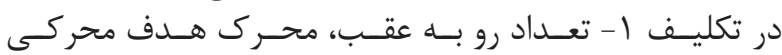

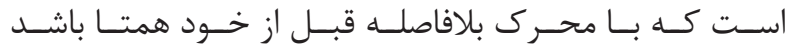

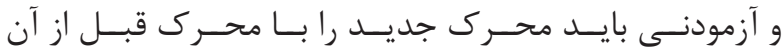

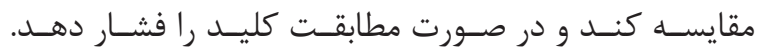

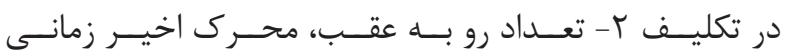

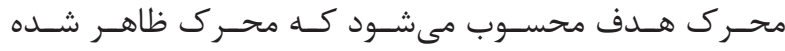

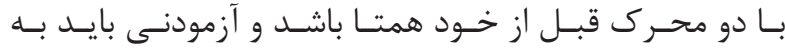

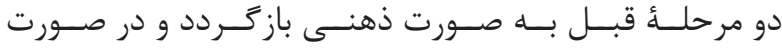

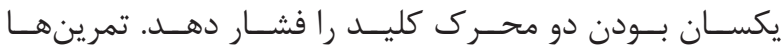

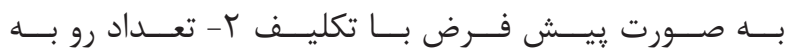

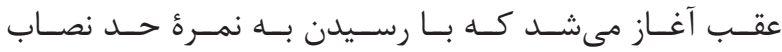

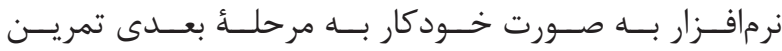

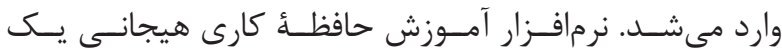

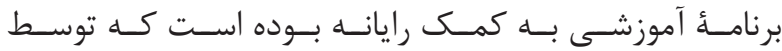

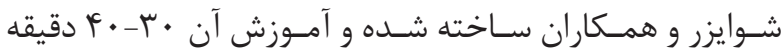

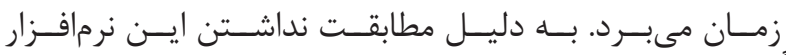

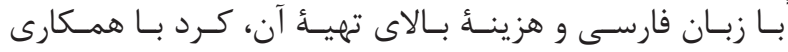

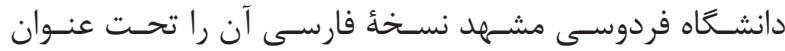

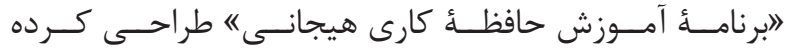

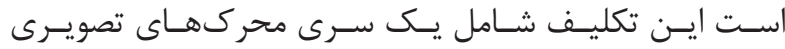

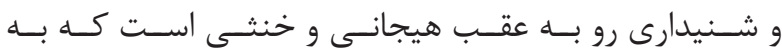

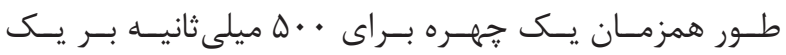

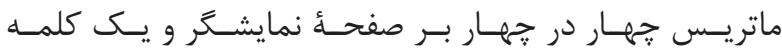

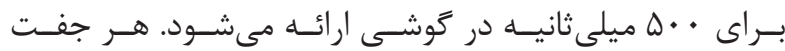

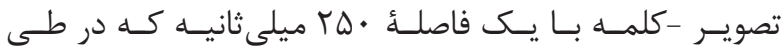

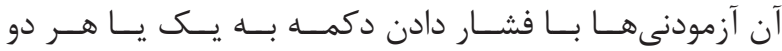

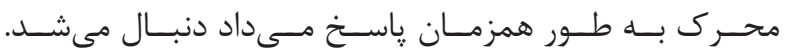

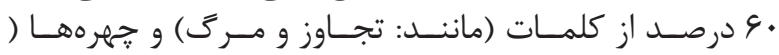

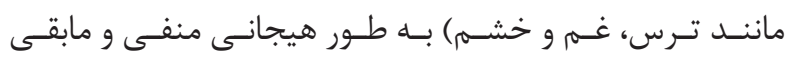


جدول ا- ميانكَين و انحراف معيار نمرات مربوط به عدم تقارن بتا بين دو مناطف پِيشانى راست و هِ.

\begin{tabular}{|c|c|c|c|}
\hline تروه كنترل (n=|n) & كروه آزمايش (19=19) & \multirow{2}{*}{ زمان } & \multirow{2}{*}{ متغير } \\
\hline ميانگين (انحراف معيار) & ميانئين (انحراف معيار) & & \\
\hline$(\Lambda F / r r) q \cdot / \Delta V$ & $(\varepsilon \cdot / 1 \vee) \wedge q / 1 \varepsilon$ & ي بيش آزمون & \multirow{2}{*}{ عدم تقارن بتا } \\
\hline$(\wedge 9 / V 9) 9 V / 9$. & $(\Lambda T / \varepsilon \cdot) \mid \mathbb{E} / \cdot$. & يَسآزمون & \\
\hline
\end{tabular}

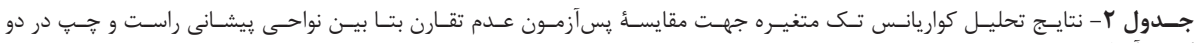

\begin{tabular}{|c|c|c|c|c|c|c|}
\hline مجذور اتاى & معنى دقارى & Fقدار F & ميانكين مجذورات & درجه & مجنموع مجذورات & شاخص آمارى \\
\hline$\cdot / V G$ & $\cdot \cdot \cdot 1$ & $1 \cdot 9 / \mathrm{dV}$ & 19FVUT/GT & 1 & 19TVQT/GT & اثر بيش آزمون \\
\hline$\cdot r \Delta$ & $\cdot \cdot \cdot r$ & $11 / \pi r$ & $r . .19 / 14$ & 1 & $r . . / q / / F$ & اثر عضويت كُروهى \\
\hline
\end{tabular}

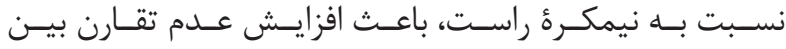

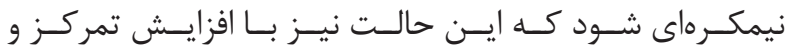

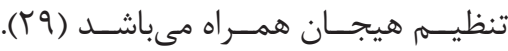

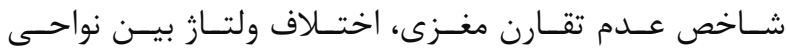

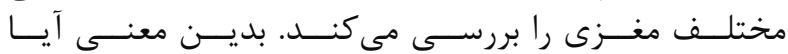

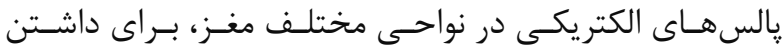

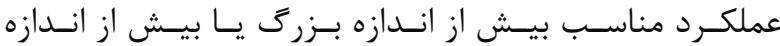

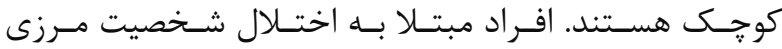

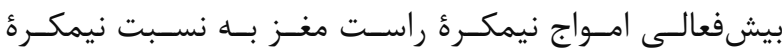

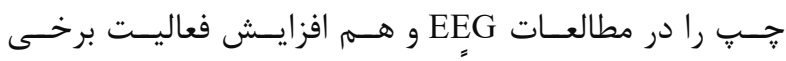

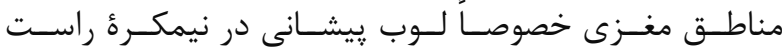

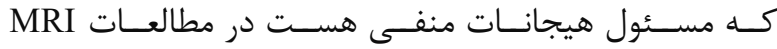

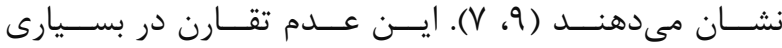

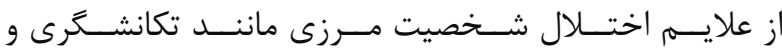

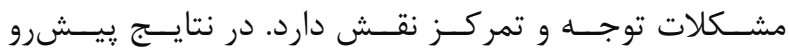

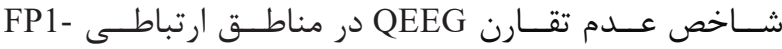

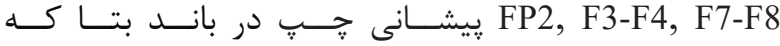

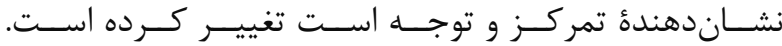

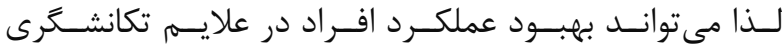

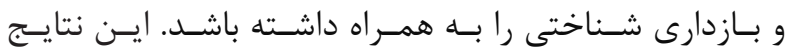

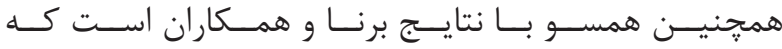

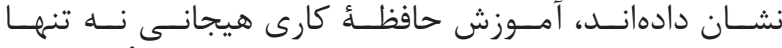

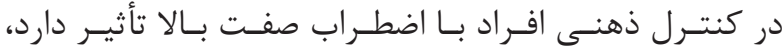

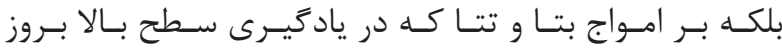

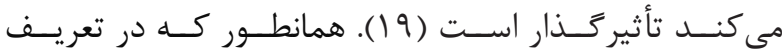

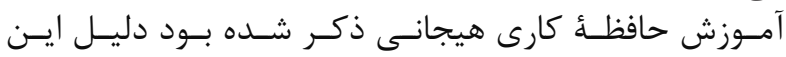

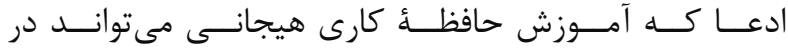

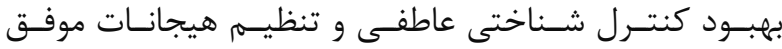

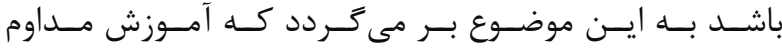

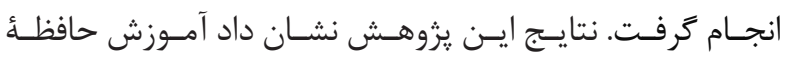

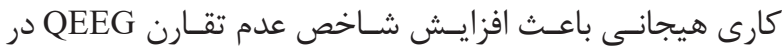

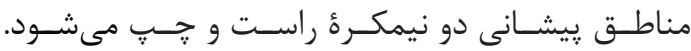

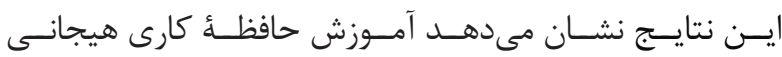

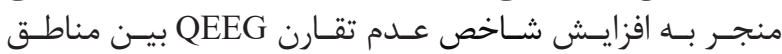

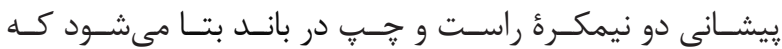

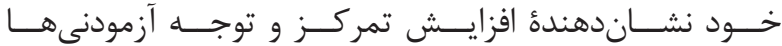

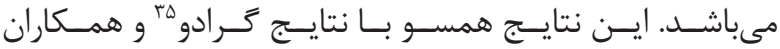

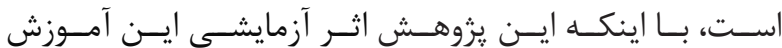

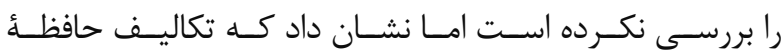

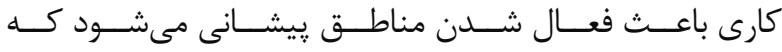

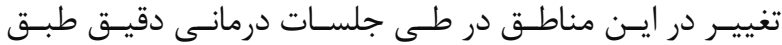

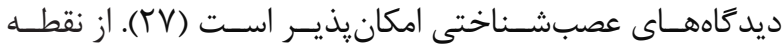

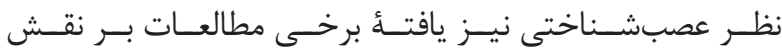

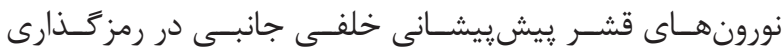

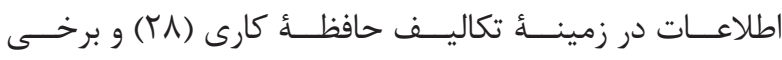

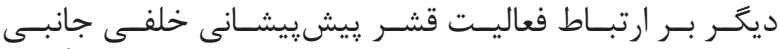

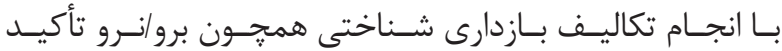

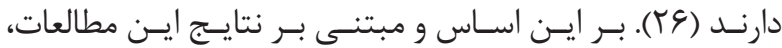

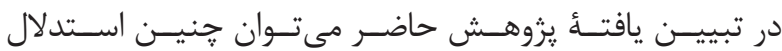

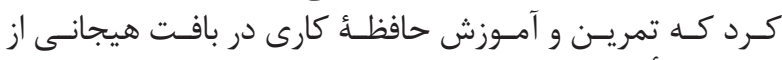

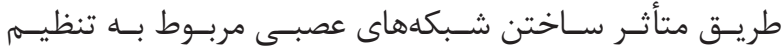

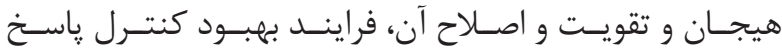

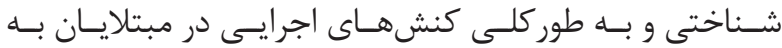

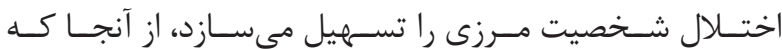

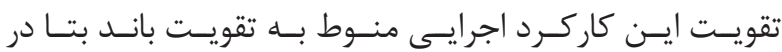

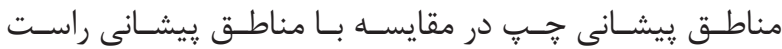

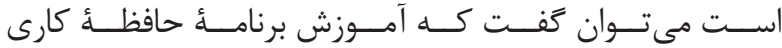

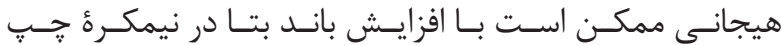

${ }^{35}$ Garrido 


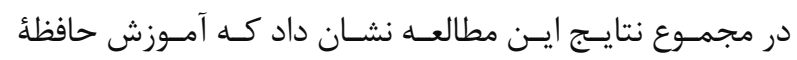

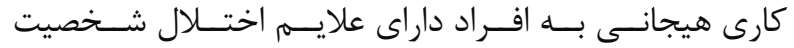

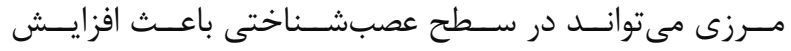

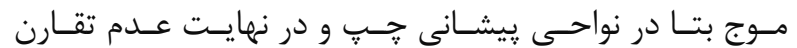

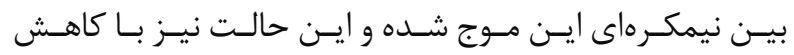

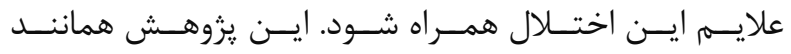

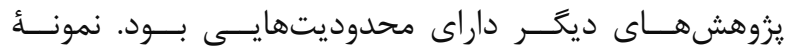

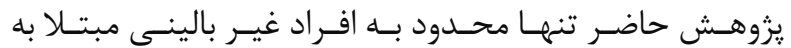

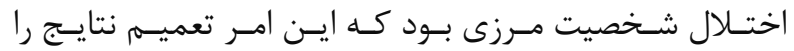

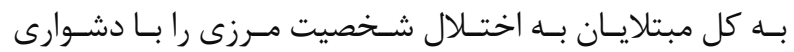

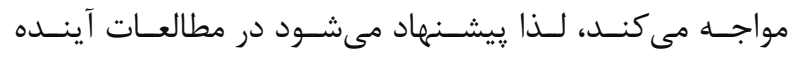

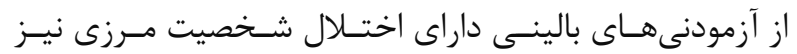

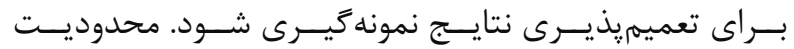

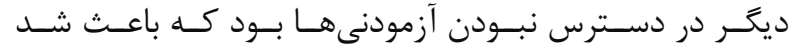

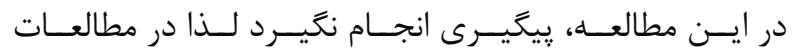

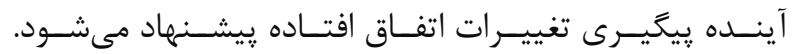

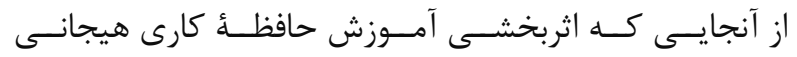

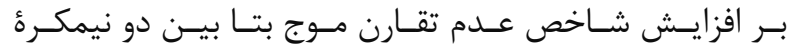

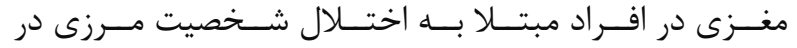

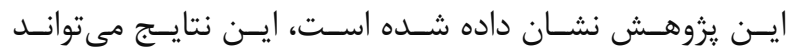

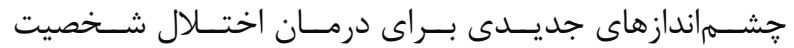

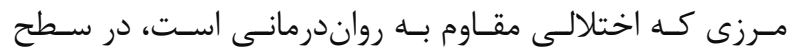
عصبشــناختى بخشـــايد. تشكر و قدردانى

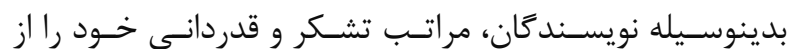

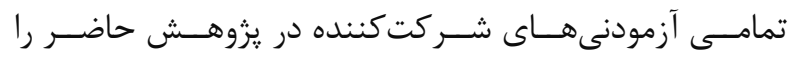

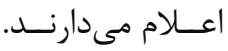

1. Association AP. Diagnostic and statiscal manual of Mental disorder. $5^{\text {th }}$ ed. 2013.

2. Salehi M, Malekian A, Haghighi M, Jahangard L, Rahimi E. Personality disorders in treatment-seeking substance dependent patients. Journal of Psychiatry and Behavioral Sciences. 2008; 2(2): 10-4.

3. Sadeghi K, Saberi SM, Assareh M. Epidemiological study of psychiatric disorder in Kermanshah urban residents. Journal of Psychiatry and Clinical Psychology. 2000; 6(2): 16-26.

4. Andrulonis PA, Glueck BC, Stroebel CF, Vogel NG, Shapiro AL, Aldridge DM. Organic brain dysfunction and the borderline syndrome. Psychiatr Clin North Am. 1981; 4(1): 47-66.

5. Banich MT, Compton RJ. Cognitive neuroscience. Ardabil: University of Mohaghegh Ardebil University Press; 2011.

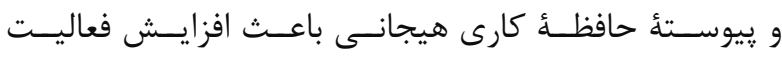

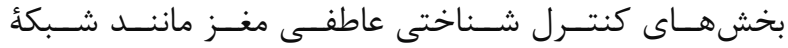

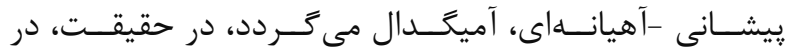

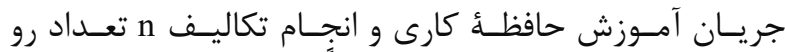

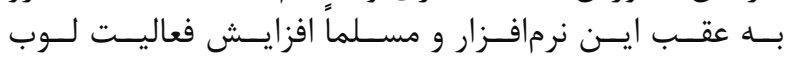

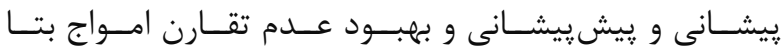

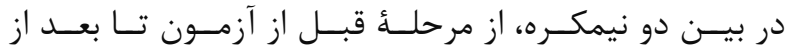

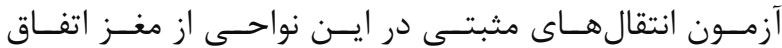

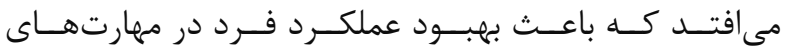

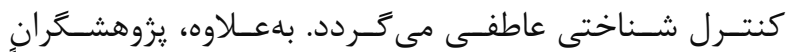

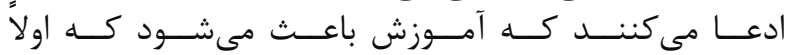

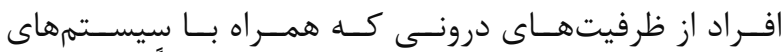

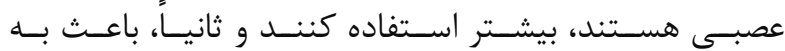

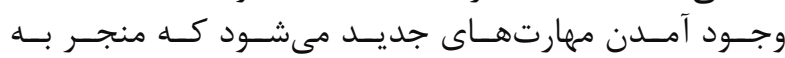

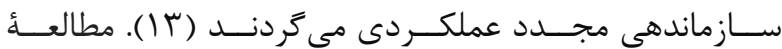

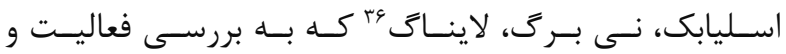

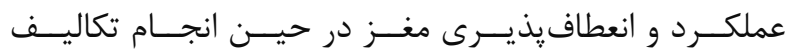

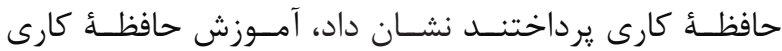

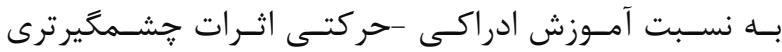

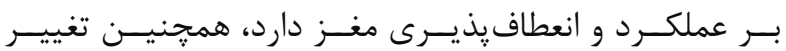

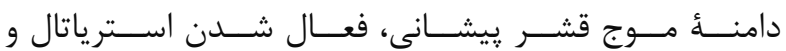
DLPF ،VLPFC

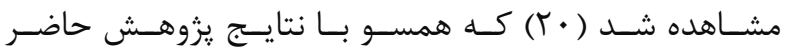

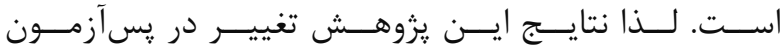

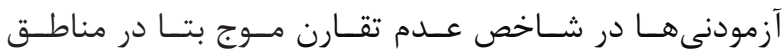

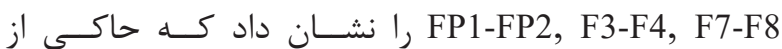

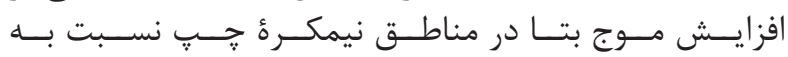

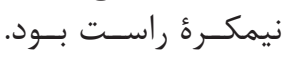

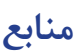

6. De la Fuente JM, Bengoetxea E, Navarro F, Bobes $\mathrm{J}$, Alarcón RD. Interconnection between biological abnormalities in borderline personality disorder: use of the Bayesian networks model. Psychiatry Research. 2011; 186(2-3): 315-9.

7. de Araujo Filho, Maria G, Abdallah C, Sato JR, de Araujo TB, Lisondo CM, et al. Morphometric hemispheric asymmetry of orbitofrontal cortex in women with borderline personality disorder: A multi-parameter approach. Psychiatry Res. 2014; 223(2): 61-6.

8. Zibman S, Daniel E, Alyagon U, Etkin A, Zangen A. Interhemispheric cortico-cortical paired associative stimulation of the prefrontal cortex jointly modulates frontal asymmetry and emotional reactivity. Brain Stimul. 2019; 12(1): 139-47.

9. Flasbeck V, Popkirov S, Brüne M. Frontal EEG asymmetry in borderline personality disorder is associated with alexithymia. Borderline Personal Disord

${ }^{36}$ Salmi, Nyberg, Laine 
Emot Dysregul. 2017; 4(1): 20. doi: 10.1186/s40479017-0071-7.

10. Schmahl C, Bremner JD. Neuroimaging in borderline personality disorder. J Psychiatr Res. 2006; 40(5): 419-27.

11. Brendel GR, Stern E, Silbersweig DA. Defining the neurocircuitry of borderline personality disorder: Functional neuroimaging approaches. Dev Psychopathol. 2005; 17(4): 1197-206.

12. Schweizer S, Grahn J, Hampshire A, Mobbs D, Dalgleish T. Training the emotional brain: improving affective control through emotional working memory training. J Neurosci. 2013; 33(12): 5301-11.

13. Mammarella N, Borella E, Carretti B, Leonardi G, Fairfield B. Examining an emotion enhancement effect in working memory: Evidence from age-related differences. Neuropsychol Rehabil. 2013; 23(3): 416-28.

14. Duncan J. The multiple-demand (MD) system of the primate brain: mental programs for intelligent behaviour. Trends Cogn Sci. 2010; 14(4): 172-9.

15. Brass M, Ullsperger M, Knoesche TR, Cramon DYv, Phillips NA. Who comes first? The role of the prefrontal and parietal cortex in cognitive control. J Cogn Neurosci. 2005; 17(9): 1367-75.

16. Owen AM, McMillan KM, Laird AR, Bullmore E. N-back working memory paradigm: A meta-analysis of normative functional neuroimaging studies. Hum Brain Mapp. 2005; 25(1): 46-59.

17. Price JL, Drevets WC. Neural circuits underlying the pathophysiology of mood disorders. Trends Cogn Sci. 2012; 16(1): 61-71.

18. Schweizer S, Dalgleish T. The impact of affective contexts on working memory capacity in healthy populations and in individuals with PTSD. Emotion. 2016; 16(1): 16-23.

19. Sari BA, Koster EH, Pourtois G, Derakshan N. Training working memory to improve attentional control in anxiety: A proof-of-principle study using behavioral and electrophysiological measures. Biol Psychol. 2016; 121: 203-12.

20. Salmi J, Nyberg L, Laine M. Working memory training mostly engages general-purpose large-scale networks for learning. Neurosci Biobehav Rev. 2018; 93: $108-22$.

21. Jackson M, Claridge G. Reliability and validity of a psychotic traits questionnaire (STQ). Br J Clin Psychol. 1991; 30(4): 311-23.

22. Rawlings D, Claridge G, Freeman JL. Principal components analysis of the schizotypal personality scale (STA) and the borderline personality scale (STB). Personality and Individual Differences. 2001; 31(3): 409-19.

23. Mohammadzadeh A, Goodarzi MA, Taghavi MR, Malazadeh J. Investigating the factor structure, validity, reliability, and normalization of the border characteristics scale in Shiraz university students. The Quarterly Journal of Fundamentals of Mental Health. 2005; 28: 75-98.

24. First M, Spitzer R, Giben M, Wiliams J. Semistructred by cilinical interview for disorder of DSM-IV-TR. Tehran: Danzhe; 2010.

25. Kord Tamini M. The effectiveness of emotional work memory training on cognitive and emotional control and emotional regulation in people with high trait anxiety (MSc). Mashhad: Ferdowsi University of Mashhad; 2015.

26. Samimi Z, Hasani J, Kord M, Afzoon J. Effectiveness of emotional work memory training on the ability of cognitive and emotional control in adolescents with post traumatic stress disorder. Journal of Mental Health. 2016; 2(4): 21-37.

27. González-Garrido AA, López-Franco AL, GómezVelázquez FR, Ramos-Loyo J, Sequeira H. Emotional content of stimuli improves visuospatial working memory. Neurosci Lett. 2015; 585: 43-7.

28. León-Domínguez U, Martín-Rodríguez JF, León-Carrión J. Executive n-back tasks for the neuropsychological assessment of working memory. Behav Brain Res. 2015; 292: 167-73.

29. Basharpoor S, Amani S, Rashid S. The relationship between QEEG waves as a neurophysiological markers in FP1 / Fp2 areas and multiple aspects of impulsivity. International Journal of High Risk Behaviours and Addiction. 2018; 7(3): 537-86. 